\title{
3-COLORING AND \\ OTHER ELEMENTARY INVARIANTS OF KNOTS
}

\author{
JÓZEF H. PRZYTYCKI \\ Department of Mathematics, George Washington University, Washington, D.C. 20052, U.S.A. \\ E-mail: przytyck@math.gwu.edu
}

Classical knot theory studies the position of a circle (knot) or of several circles (link) in $R^{3}$ or $S^{3}=R^{3} \cup \infty$. The fundamental problem of classical knot theory is the classification of links (including knots) up to the natural movement in space which is called an ambient isotopy. To distinguish knots or links we look for invariants of links, that is, properties of links which are unchanged under ambient isotopy. When we look for invariants of links we have to take into account the following three criteria:

1. Is our invariant easy to compute?

2. Is it easy to distinguish elements in the value set of the invariant?

3. Is our invariant good at distinguishing links?

The number of components of a link, $\operatorname{com}(L)$, is the simplest invariant. A more interesting link invariant is given by the linking number, defined in 1833 by C. F. Gauss using a certain double integral [Ga]. H. Brunn noted in 1892 that the linking number has a simple combinatorial definition $[\mathrm{Br}]$.

Definition 0.1. Let $D$ be an oriented link diagram. Each crossing has an associated sign: +1 for 1 and -1 for The global linking number of $D, l k(D)$, is defined to be half of the sum of the signs of crossings between different components of the link diagram. If the diagram has no crossings, we put $l k(D)=0$.

To show that a function defined on diagrams of links is an invariant of (global isotopy) of links, we have to interpret global isotopy in terms of diagrams. This was done by Reidemeister [Re, 1927] and Alexander and Briggs [A-B, 1927].

1991 Mathematics Subject Classification: 57M.

Supported by USAF grant 1-443964-22502 while visiting the Mathematics Department, U.C. Berkeley.

The paper is in final form and no version of it will be published elsewhere. 
ThEOREM 0.2 (Reidemeister theorem). Two link diagrams are ambient isotopic if and only if they are connected by a finite sequence of Reidemeister moves $R_{i}^{ \pm 1}, i=1,2,3$ (see Fig. 0.1) and isotopy (deformation) of the plane of the diagram. The theorem holds also for oriented links and diagrams. One then has to take into account all possible coherent orientations of diagrams involved in the moves.
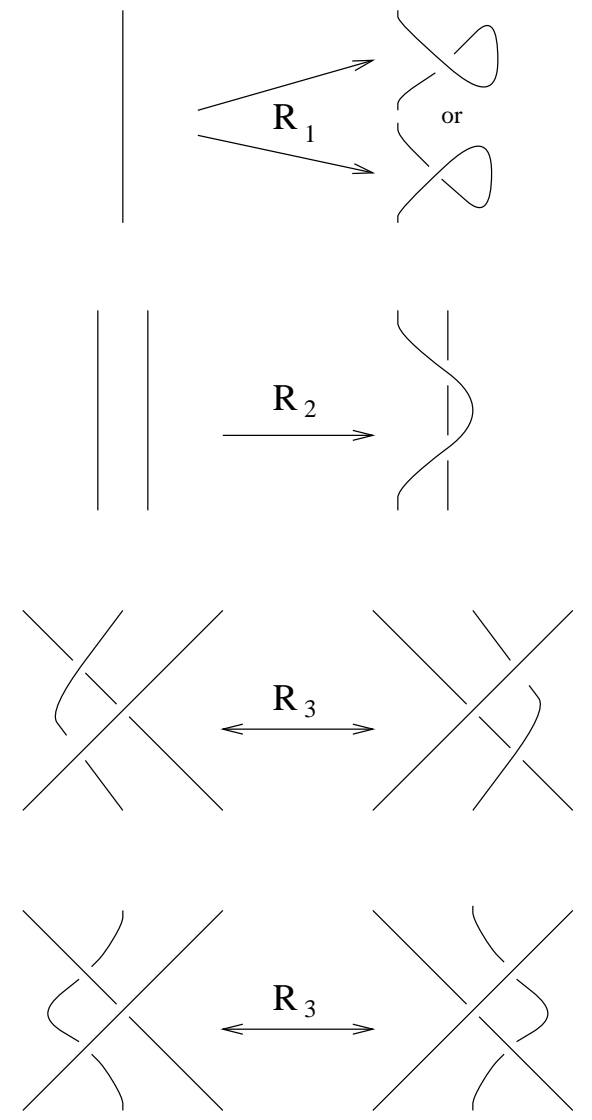

Fig. 0.1

EXERCISE 0.3. Show that $l k(D)$ is preserved by Reidemeister moves on oriented link diagrams. Thus $l k$ is an invariant of oriented links.

ExAmple 0.4. $l k(\bigcirc \bigcirc)=0, l k(\forall)=1, l k(1)=-1$. Therefore the global linking number allows us to distinguish the trivial link of two components, $T_{2}$, the righthanded Hopf link, $2_{1}$, and the left-handed Hopf link, $\overline{2}_{1}$.

1. The tricoloring. The tricoloring invariant (or 3-coloring) is the simplest invariant which distinguishes between the trefoil knot and the trivial knot. The idea of tricoloring was introduced by R.Fox around 1960, [C-F, Chapter VI, Exercises 6-7], [F-2], and has been extensively used and popularized by J. Montesinos [Mon] and L. Kauffman [K]. 
Definition $1.1([\mathrm{P}-1])$. We say that a link diagram $D$ is tricolored if every arc is colored $r$ (red), $b$ (blue) or $y$ (yellow) (we consider arcs of the diagram literally, so that in the undercrossing one arc ends and the second starts; compare Fig. 1.1), and at any given crossing either all three colors appear or only one color appears. The number of different tricolorings is denoted by $\operatorname{tri}(D)$. If a tricoloring uses only one color we say that it is a trivial tricoloring.

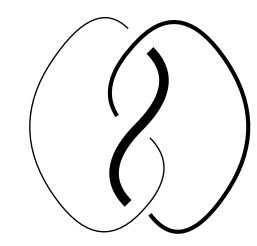

Fig. 1.1. Different colors are marked by lines of different thickness.

LEMMA 1.2. The tricoloring is an (ambient isotopy) link invariant.

Proof. We have to check that $\operatorname{tri}(D)$ is preserved under the Reidemeister moves. The invariance under $R_{1}$ and $R_{2}$ is illustrated in Fig. 1.2 and the invariance under $R_{3}$ is illustrated in Fig. 1.3.
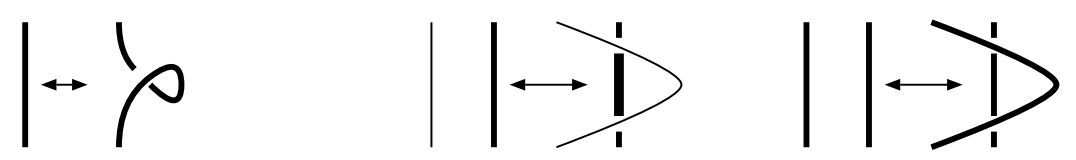

Fig. 1.2
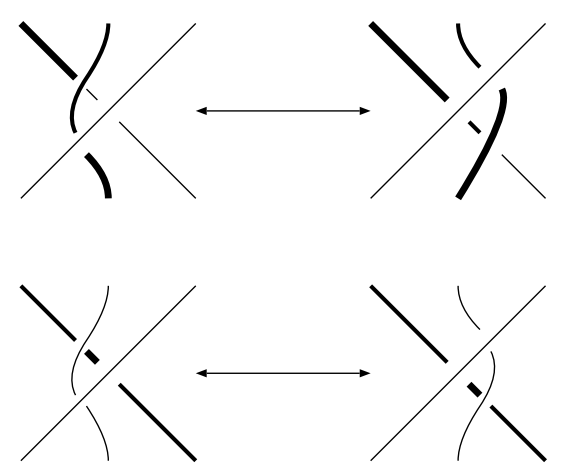

Fig. 1.3

Because the trivial knot has only trivial tricolorings, $\operatorname{tri}\left(T_{1}\right)=3$, and the trefoil knot allows a nontrivial tricoloring (Fig. 1.1), it follows that the trefoil knot is a nontrivial knot.

ExERCISE 1.3. Find the number of tricolorings for the trefoil knot $\left(3_{1}\right)$, the figure eight knot $\left(4_{1}\right)$ and the square knot $\left(3_{1} \# \overline{3}_{1}\right.$, see Fig. 1.4). Then deduce that these knots are pairwise different. 
LEMMA 1.4. $\operatorname{tri}(L)$ is always a power of 3 .

Pro of. Denote the colors by 0,1 and 2 and treat them modulo 3, that is, as elements of the group (field) $Z_{3}$. All colorings of the arcs of a diagram using colors $0,1,2$ (not necessarily allowed 3-colorings) can be identified with the group $Z_{3}^{r}$ where $r$ is the number of arcs of the diagram. The (allowed) 3-colorings can be characterized by the property that at each crossing the sum of the colors is equal to zero modulo 3. Thus (allowed) 3-colorings form a subgroup of $Z_{3}^{r}$.

The elementary properties of tricolorings, which we give in Lemma 1.5, follow immediately from the connections between tricolorings and the Jones and Kauffman polynomials of links. We will give here an elementary proof of (a)-(c) of Lemma 1.5. There is also an elementary proof of (d) (based on the flow-potential idea of Jaeger, see [Ja-P]), but it is more involved; compare Lemma 2.2 .

LEMMA 1.5. (a) tri $\left(L_{1}\right) \operatorname{tri}\left(L_{2}\right)=3 \operatorname{tri}\left(L_{1} \# L_{2}\right)$, where \# denotes the connected sum of links (see Fig. 1.4).

(b) Let $L_{+}, L_{-}, L_{0}$ and $L_{\infty}$ denote four unoriented link diagrams as in Fig. 1.5. Then, among four numbers tri $\left(L_{+}\right), \operatorname{tri}\left(L_{-}\right), \operatorname{tri}\left(L_{0}\right)$ and $\operatorname{tri}\left(L_{\infty}\right)$, three are equal one to another and the fourth is equal to them or is 3 times bigger.

In particular:

(c) $\operatorname{tri}\left(L_{+}\right) / \operatorname{tri}\left(L_{-}\right)=1$ or 3 , or $1 / 3$.

Part (b) can be strengthened to show that:

(d) Not all the numbers tri $\left(L_{+}\right), \operatorname{tri}\left(L_{-}\right), \operatorname{tri}\left(L_{0}\right)$ and $\operatorname{tri}\left(L_{\infty}\right)$ are equal.

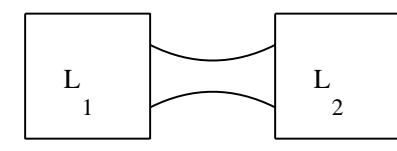

$\mathrm{L}_{1} \mathrm{HL}_{2}$

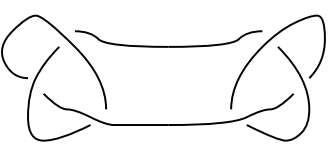

$3_{1} \# \overline{3}_{1}$

Fig. 1.4

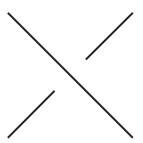

$\mathrm{L}+$

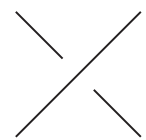

$\mathrm{L}_{-}$

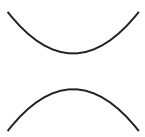

$\mathrm{L}_{0}$

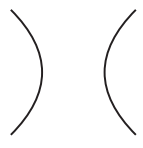

$\mathrm{L} \infty$

Fig. 1.5

P r o of. (a) An $n$-tangle is a part of a link diagram placed in a 2-disk, with $2 n$ points on the disk boundary ( $n$ inputs and $n$ outputs); Fig. 1.6. We show first that for any 3-coloring of a 1-tangle (i.e. a tangle with one input and one output; see Fig. 1.6(a)), the input arc have the same color as the output arc. Namely, let $T$ be our 3-colored tangle and let the 1-tangle $T^{\prime}$ be obtained from $T$ by adding a trivial component, $C$, below $T$, 
close to the boundary of the tangle, so that it cuts $T$ only near the input and the output; Fig. 1.6(b). Of course the 3-coloring of $T$ can be extended to a 3-coloring of $T^{\prime}$ (in three different ways), because the tangle $T^{\prime}$ is ambient isotopic to a tangle obtained from $T$ by adding a small trivial component disjoint from $T$. If we, however, try to color $C$, we see immediately that it is possible iff the input and the output $\operatorname{arcs}$ of $T$ have the same color.

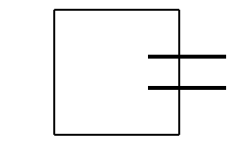

(a)

T

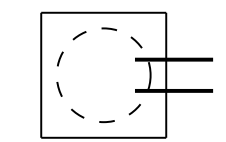

(b) $\mathrm{T}^{\prime}$

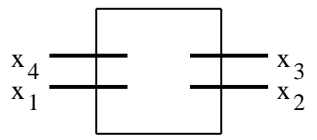

(c)

$\mathrm{T}_{\mathrm{D}}$

Fig. 1.6

Thus if we consider a connected sum $L_{1} \# L_{2}$, we see from the above that the $\operatorname{arcs}$ joining $L_{1}$ and $L_{2}$ have the same color. Therefore the formula, $\operatorname{tri}\left(L_{1}\right) \operatorname{tri}\left(L_{2}\right)=$ $3 \operatorname{tri}\left(L_{1} \# L_{2}\right)$, follows.

(b) Consider a crossing $p$ of the diagram $D$. If we cut out of $D$ a neighborhood of $p$, we are left with the 2-tangle, $T_{D}$ (see Fig. 1.6(c)). The set of 3-colorings of $T_{D}$, $\operatorname{Tr} i\left(T_{D}\right)$, forms a $Z_{3}$ linear space. Each of the sets of 3 -colorings of $D_{+}, D_{-}, D_{0}$ and $D_{\infty}$, $\operatorname{Tr} i\left(D_{+}\right), \operatorname{Tr} i\left(D_{-}\right), \operatorname{Tr} i\left(D_{0}\right)$ and $\operatorname{Tr} i\left(D_{\infty}\right)$, respectively form a subspace of $\operatorname{Tr} i\left(T_{D}\right)$. Let $x_{1}, x_{2}, x_{3}, x_{4}$ be generators of $\operatorname{Tr} i\left(T_{D}\right)$ corresponding to arcs cutting the boundary of the tangle; see Fig. 1.6(c). Then any element of $\operatorname{Tri}\left(T_{D}\right)$ satisfies the equality $x_{1}-x_{2}+$ $x_{3}-x_{4}=0$. To show this, we proceed as in part (a). Any element of $\operatorname{Tri}\left(D_{+}\right)$(resp. $\operatorname{Tr} i\left(D_{-}\right)$, $\operatorname{Tr} i\left(D_{0}\right)$ and $\operatorname{Tr} i\left(D_{\infty}\right)$ ) satisfies additionally the equation $x_{2}=x_{4}$ (resp. $x_{1}=x_{3}, x_{1}=x_{2}$ and $\left.x_{1}=x_{4}\right)$. Thus $\operatorname{Tri}\left(D_{+}\right)\left(\right.$resp. $\operatorname{Tri}\left(D_{-}\right), \operatorname{Tri}\left(D_{0}\right)$ and $\left.\operatorname{Tr}\left(D_{\infty}\right)\right)$ is a subspace of $\operatorname{Tr} i\left(T_{D}\right)$ of codimension at most one. Let $F$ be the subspace of $\operatorname{Tr} i\left(T_{D}\right)$ given by the equations $x_{1}=x_{2}=x_{3}=x_{4}$, that is the space of 3 -colorings monochromatic on the boundary of the tangle. $F$ is a subspace of codimension at most one in any of the spaces $\operatorname{Tr} i\left(D_{+}\right), \operatorname{Tr} i\left(D_{-}\right), \operatorname{Tr} i\left(D_{0}\right), \operatorname{Tr} i\left(D_{\infty}\right)$. Furthermore the common part of any two of $\operatorname{Tr} i\left(D_{+}\right), \operatorname{Tr} i\left(D_{-}\right), \operatorname{Tr} i\left(D_{0}\right), \operatorname{Tr} i\left(D_{\infty}\right)$ is equal to $F$. To see this we just compare the defining relations for these spaces. Finally notice that $\operatorname{Tri}\left(D_{+}\right) \cup \operatorname{Tr} i\left(D_{-}\right) \cup \operatorname{Tr} i\left(D_{0}\right) \cup$ $\operatorname{Tri}\left(D_{\infty}\right)=\operatorname{Tri}\left(T_{D}\right)$.

We have the following possibilities:

(1) $F$ has codimension 1 in $\operatorname{Tri}\left(T_{D}\right)$ Then by the above considerations one of $\operatorname{Tr} i\left(D_{+}\right)$, $\operatorname{Tr} i\left(D_{-}\right), \operatorname{Tr} i\left(D_{0}\right), \operatorname{Tr} i\left(D_{\infty}\right)$ is equal to $\operatorname{Tr} i\left(T_{D}\right)$. The remaining three spaces are equal to $F$ and (d) (thus also (b)) of Lemma 1.5 holds.

(2) $F=\operatorname{Tri}\left(D_{+}\right)=\operatorname{Tri}\left(D_{-}\right)=\operatorname{Tr} i\left(D_{0}\right)=\operatorname{Tri}\left(D_{\infty}\right)=\operatorname{Tri}\left(T_{D}\right)$.

(3) $F$ has codimension 2 in $\operatorname{Tri}\left(T_{D}\right)$. Then $3|F|=\operatorname{tri}\left(D_{+}\right)=\operatorname{tri}\left(D_{-}\right)=\operatorname{tri}\left(D_{0}\right)=$ $\operatorname{tri}\left(D_{\infty}\right)=\frac{1}{3} \operatorname{tri}\left(T_{D}\right)$

This completes the proof of (b) and (c) of Lemma 1.5. To complete (d) of Lemma 1.5 one must exclude cases (2) and (3). This can be done by showing that tricolorings can be interpreted via the so called Goeritz matrix of the link diagram; compare Lemma 2.2 and see $[\mathrm{J}-\mathrm{P}]$. 
Part (c) of Lemma 1.5 can be used to approximate the unknotting (Gordian) number of a knot, $u(K)$; compare [Mur].

COROLlaRY 1.6. $u(K) \geq \log _{3}(\operatorname{tri}(K))-1$. In particular for the square knot: $u\left(3_{1} \# \overline{3}_{1}\right)$ $=2$.

COROllary 1.7. If $L$ is a link with $k$-bridge presentation ${ }^{1}$ then $\operatorname{tri}(L) \leq 3^{k}$.

I noticed the connection between tricolorings and the Jones polynomial when I analyzed the influence of 3 -moves on the 3 -coloring and the Jones polynomial [P-1].

Definition 1.8. The local change in a link diagram which replaces parallel lines by $n$ positive half-twists is called an $n$-move; see Fig. 1.7.

Lemma 1.9. Let the diagram $D_{+++}$be obtained from $D$ by a 3-move (Fig. 1. $\left.7(a)\right)$. Then:

(a) $\operatorname{tri}\left(D_{+++}\right)=\operatorname{tri}(D)$,

(b) $V_{D_{+++}}\left(e^{2 \pi i / 6}\right)= \pm i^{\left(\operatorname{com}\left(D_{+++}\right)-\operatorname{com}(D)\right)} V_{D}\left(e^{2 \pi i / 6}\right)$, where $V$ is the Jones polynomial,

(c) $F_{D_{+++}}(1,-1)=F_{D}(1,-1)$, where $F$ is the Kauffman polynomial.

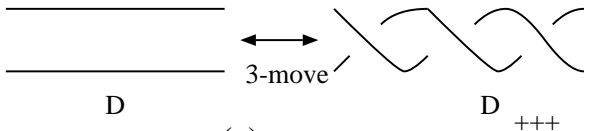

(a)

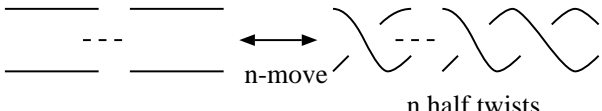

(b)

Fig. 1.7

Proof. We prove (a) and (c) leaving (b) as an exercise.

(a) The bijection between 3-colorings of $D$ and $D_{+++}$is illustrated in Fig. 1.8.

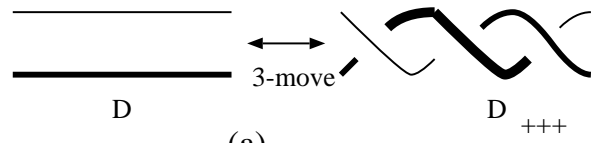

(a)

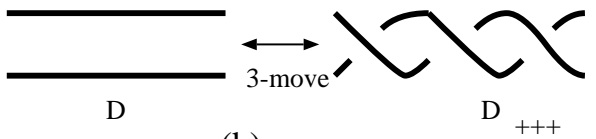

(b)

Fig. 1.8

(c) $F_{D_{+++}}(1,-1)=-F_{D_{+}}(1,-1)-F_{D_{++}}(1,-1)-F_{D_{\infty}}(1,-1)=-F_{D_{+}}(1,-1)+$ $F_{D}(1,-1)+F_{D_{+}}(1,-1)+F_{D_{\infty}}(1,-1)-F_{D_{\infty}}(1,-1)=F_{D}(1,-1)$.

One can easily check that for a trivial $n$-component link, $T_{n}, \operatorname{tri}\left(T_{n}\right)=$ $3^{n}=3 V_{T_{n}}^{2}\left(e^{2 \pi i / 6}\right)=3(-1)^{n-1} F_{T_{n}}(1,-1)$. Furthermore it follows from Lemma 1.9 that as long as a link $L$ can be obtained from a trivial link by 3 -moves we have: $\operatorname{tri}(L)=$ $3\left|V_{L}^{2}\left(e^{2 \pi i / 6}\right)\right|=3\left|F_{L}(1,-1)\right|$.

\footnotetext{
${ }^{1}$ Let $L$ be a link in $R^{3}$ which meets a plane $E \subset R^{3}$ in $2 k$ points such that the arcs of $L$ contained in each halfspace relative to $E$ possess orthogonal projections onto $E$ which are simple and disjoint. $(L, E)$ is called a $k$-bridge presentation of $L$; [B-Z].
} 
It may look strange that such a natural problem, whether any link can be reduced to an unlink by 3 -moves is an open problem.

Conjecture 1.10 (Montesinos-Nakanishi). Any link can be reduced to a trivial link by a sequence of 3-moves.

Rem ark 1.11. Nakanishi first considered the conjecture in 1981. Montesinos analyzed 3-moves before, in connection with 3-fold dihedral branch coverings, and asked a related but different question. Conjecture 1.10 holds for algebraic links (in the Conway sense). It would be a "finite" check whether conjecture holds for links with braid index at most 5 (and bridge index at most 3) as Coxeter (1957) showed that the quotient of the braid group $B_{n} /<\sigma_{1}^{3}>$ is finite for $n \leq 5$.

According to Nakanishi (1994) the smallest known obstruction to the conjecture is the 2-parallel of the Borromean rings (notice that it is a 6 -string braid), Fig. 1.9.

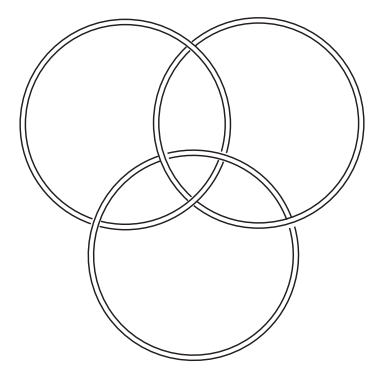

Fig. 1.9

Lemma 1.5 suggests the following stronger conjecture.

Conjecture 1.12. Any 2-tangle can be reduced, using 3-moves, to one of the four 2-tangles of Figure 1.10. We allow additionally trivial components in the tangles of Fig. 1.10 .

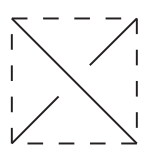

$\mathrm{S}_{+}$

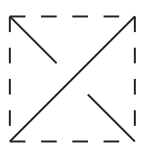

$\mathrm{S}_{-}$

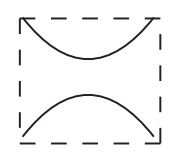

$\mathrm{S}_{0}$

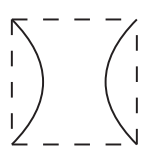

$S \infty$

Fig. 1.10

Conjecture 1.10 suggests that the formula linking tricoloring with the Jones and Kauffman polynomials holds for any link. This is, in fact, the case.

THEOREM 1.13. (a) $\operatorname{tri}(L)=3\left|V_{L}^{2}\left(e^{2 \pi i / 6}\right)\right|$.

(b) $\operatorname{tri}(L)=3\left|F_{L}(1,-1)\right|$.

The proof of (a) in [P-1] uses Fox's interpretation of 3-coloring and the connection with the first homology group of the branched 2-fold cover of $S^{3}$ branched over the link. Now however we can give totally elementary proof based on Lemma 1.5(d). 
Proof. Because $\operatorname{tri}(L)$ is a power of 3 , we can consider the signed version of the tricoloring defined by: $\operatorname{tri}^{\prime}(L)=(-1)^{\log _{3}(\operatorname{tri}(L))} \operatorname{tri}(L)$. It follows from Lemma 1.5 (d) that

$$
\operatorname{tri}^{\prime}\left(L_{+}\right)+\operatorname{tri} i^{\prime}\left(L_{-}\right)=-\operatorname{tri} i^{\prime}\left(L_{0}\right)-\operatorname{tri} i^{\prime}\left(L_{\infty}\right) .
$$

This is however exactly the recursive formula for the $\operatorname{Kauffman}$ polynomial $F_{L}(a, x)$ at $(a, x)=(1,-1)$. Comparing the initial data (for the unknot) of $\operatorname{tri}^{\prime}$ and $F(1,-1)$ we get generally that: $-3 F_{L}(1,-1)=\operatorname{tri}^{\prime}(L)=(-1)^{\log _{3}(\operatorname{tri}(L))} \operatorname{tri}(L)$, which proves part (b) of Theorem 1.13. Part (a) follows from Lickorish's observation [Li], that $F_{L}(1,-1)=$ $(-1)^{\operatorname{com}(L)} V^{2}\left(e^{2 \pi i / 6}\right)$.

The value of the Jones polynomial $V_{L}\left(e^{2 \pi i / 6}\right)$ is a slightly more delicate invariant than the tricoloring, $\operatorname{tri}(L)$, or $F_{L}(1,-1)$ (essentially it is just a "sign"). P. Traczyk has given, however, an idea which allows us to utilize this sign to approximate the unknotting number in a better way than in Corollary 1.6.

TheOREM 1.14. Let $r(L)=\log _{3}\left|V_{L}^{2}\left(e^{2 \pi i / 6}\right)\right|\left(=\log _{3}(\operatorname{tri}(L))-1\right)$. If a knot $K$ can be trivialized by changing $r(K)$ crossings and $V_{K}\left(e^{2 \pi i / 6}\right)=\epsilon_{K}(i \sqrt{3})^{r(K)}$, where $\epsilon_{K}= \pm 1$, then the number of negative crossings, which are changed, is congruent to $l\left(\epsilon_{K}\right)$ modulo 2 , where $(-1)^{l\left(\epsilon_{K}\right)}=\epsilon_{K}$.

Proof. Let $t^{1 / 2}=-e^{2 \pi i / 12}$, then for any link $L: V_{L}\left(e^{2 \pi i / 6}\right)=\epsilon_{L} i^{\operatorname{com}(L)-1}(i \sqrt{3})^{r(L)}$. Consider a pair of oriented links $L_{+}$and $L_{-}$. From the skein relation of the Jones polynomial, one gets:

$$
\begin{aligned}
\frac{1}{2}\left((1-i \sqrt{3}) \epsilon_{L_{+}} i^{\operatorname{com}\left(L_{+}\right)-1}(i \sqrt{3})^{r\left(L_{+}\right)}-(1+i \sqrt{3}) \epsilon_{L_{-}}\right. & \left.i^{\operatorname{com}\left(L_{-}\right)-1}(i \sqrt{3})^{r\left(L_{-}\right)}\right) \\
& =-i \epsilon_{L_{0}} i^{\operatorname{com}\left(L_{0}\right)-1}(i \sqrt{3})^{r\left(L_{0}\right)} .
\end{aligned}
$$

One can see immediately that the above equation cannot hold for $\left|r\left(L_{+}\right)-r\left(L_{-}\right)\right| \geq 2$. For $r\left(L_{+}\right)-r\left(L_{-}\right)=1$ it simplifies to

$$
\frac{1}{2}\left((3+i \sqrt{3}) \epsilon_{L_{+}}-(1+i \sqrt{3}) \epsilon_{L_{-}}\right)=(-1)^{\left(\frac{\operatorname{com}\left(L_{0}\right)-\operatorname{com}\left(L_{+}\right)-1}{2}\right)} \epsilon_{L_{0}}(i \sqrt{3})^{\left(r\left(L_{0}\right)-r\left(L_{-}\right)\right)} .
$$

This equation holds iff $\epsilon_{L_{+}}=\epsilon_{L_{-}}$. Similarly, for $r\left(L_{+}\right)-r\left(L_{-}\right)=-1$ one gets $\epsilon_{L_{+}}=$ $-\epsilon_{L_{-}}$. This completes the proof of Theorem 1.14, because for the trivial knot, $T_{1}$, $\epsilon_{T_{1}}=1$.

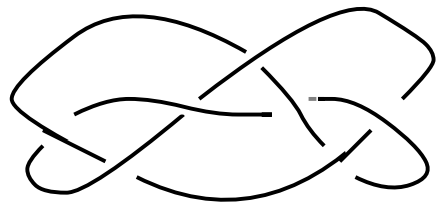

7

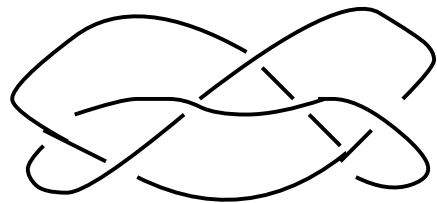

the trivial knot

Fig. 1.11 
ExAMPLES 1.15. (a) Let $K=3_{1} \# \overline{3}_{1}$, then $u(K)=2$. Furthermore if $K$ is trivialized using two crossing changes, then one positive and one negative crossing, have to be changed. Namely $V_{K}\left(e^{2 \pi i / 6}\right)=3=-(i \sqrt{3})^{2}$ and Theorem 1.14 can be used.

(b) The unknotting number of the knot $7_{7}$ is 1 ; see Fig. 1.11. However this knot cannot be trivialized by changing a positive crossing. Namely $V_{7_{7}}\left(e^{2 \pi i / 6}\right)=-i \sqrt{3}$. Notice that the signature of $7_{7}$ is equal to 0 and the Tait number of the minimal diagram is equal to +1 .

2. $n$-coloring. Tricoloring of links can be generalized, after Fox, [F-1; Chapter 6], [C-F; Chapter VIII, Exercises 8-10], [F-2], to $n$-coloring of links as follows:

Definition 2.1. We say that a link diagram $D$ is $n$-colored if every arc is colored by one of the numbers $0,1, \ldots, n-1$ in such a way that at each crossing the sum of the colors of the undercrossings is equal to twice the color of the overcrossing modulo $n$.

The following properties of $n$-colorings can be proved in a similar way as for the tricoloring properties. However, an elementary proof of the part $(\mathrm{g})$ is more involved and requires an interpretation of $\mathrm{n}$-colorings using the Goeritz matrix [Ja-P].

LEMMA 2.2. (a) Reidemeister moves preserve the number of $n$-colorings, $\operatorname{col}_{n}(D)$, thus it is a link invariant,

(b) if $D$ and $D^{\prime}$ are related by a finite sequence of $n$-moves, then $\operatorname{col}_{n}(D)=\operatorname{col}_{n}\left(D^{\prime}\right)$,

(c) $n$-colorings form an abelian group, $\operatorname{Col}_{n}(D)$,

(d) if $n$ is a prime number, then $\operatorname{col}_{n}(D)$ is a power of $n$ and for a link with b bridges: $b \geq \log _{n}\left(\operatorname{col}_{n}(L)\right)$,

(e) $\operatorname{col}_{n}\left(L_{1}\right) \operatorname{col}_{n}\left(L_{2}\right)=n\left(\operatorname{col}_{n}\left(L_{1} \# L_{2}\right)\right)$,

(f) if $n$ is a prime odd number then among the four numbers $\operatorname{col}_{n}\left(L_{+}\right), \operatorname{col}_{n}\left(L_{-}\right)$, $\operatorname{col}_{n}\left(L_{0}\right)$ and $\operatorname{col}_{n}\left(L_{\infty}\right)$, three are equal and the fourth is either equal to them or $n$ times greater,

More generally: If $L_{0}, L_{1}, \ldots, L_{n-1}, L_{\infty}$ are $n+1$ diagrams generalizing the four diagrams from $(f)$; see Fig. 2.1 then:

(g) if $n$ is a prime number then among the $n+1$ numbers $\operatorname{col}_{n}\left(L_{0}\right), \operatorname{col}_{n}\left(L_{1}\right), \ldots$, $\operatorname{col}_{n}\left(L_{n-1}\right)$ and $\operatorname{col}_{n}\left(L_{\infty}\right), n$ are equal and the $(n+1)$ th is $n$ times greater,

(h) if $n$ is a prime number, then $u(K) \geq \log _{n}\left(\operatorname{col}_{n}(K)\right)-1$.

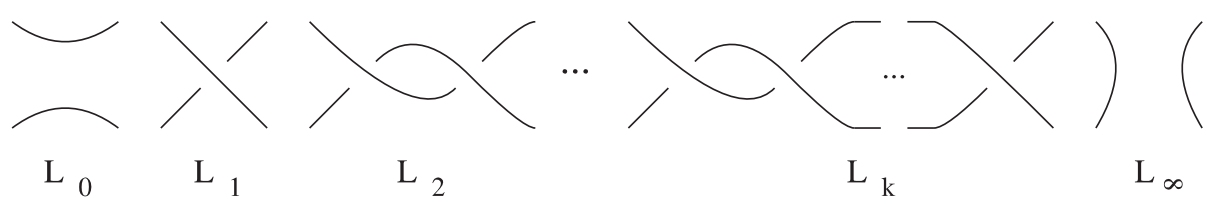

Fig. 2.1

Corollary 2.3. (i) For the figure eight knot, $4_{1}$, one has $\operatorname{col}_{5}\left(4_{1}\right)=25$, so the figure eight knot is a nontrivial knot; compare Fig. 2.2.

(ii) $u\left(4_{1} \# 4_{1}\right)=2$. 


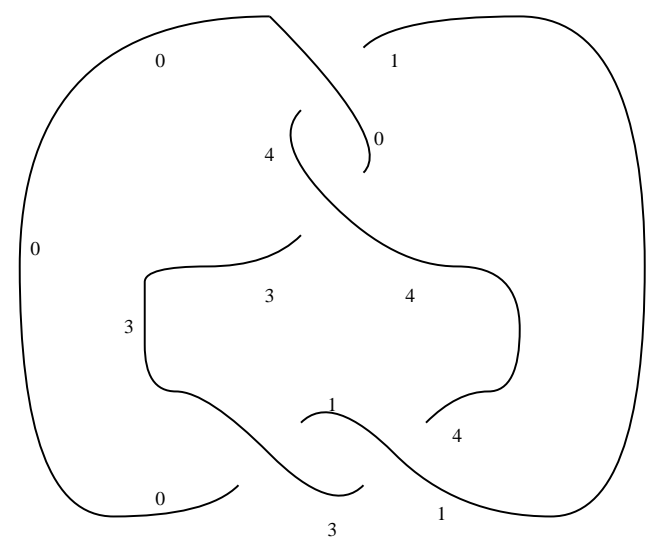

Fig. 2.2

By Lemma 2.2(b), any 5-move preserves the number of 5-colorings. On the other hand, Corollary 2.3 suggests that the move of Fig. 2.3 also preserves $\operatorname{col}_{5}(L)$.

LEMMA 2.4. If two links are related by a sequence of moves as in Fig. 2.3 (allowing the mirror image of Fig. 2.3), then they have the same number of 5-colorings.

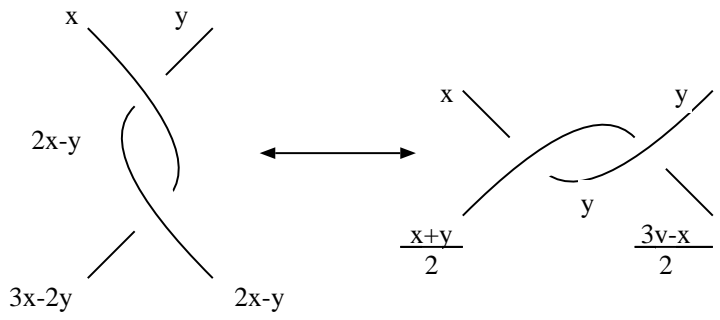

Fig. 2.3

Proof. It suffices to notice that for $x$ and $y$ of Fig. 2.3:

$$
3 x-2 y \equiv \frac{x+y}{2} \bmod 5 \quad \text { and } \quad 2 x-y \equiv \frac{3 y-x}{2} \bmod 5 .
$$

It was noticed in $[\mathrm{H}-\mathrm{U}]$ that the moves of Fig. 2.3 are more general than the 5 -moves.

Lemma $2.5([\mathrm{H}-\mathrm{U}])$. A 5-move is a combination of moves of Fig. 2.3 (and isotopy).

Proof. This is illustrated in Fig. 2.4.

It has been noticed in [P-2] that there are links which cannot be changed to trivial links using 5-moves. In particular, the figure eight knot cannot be reduced to a trivial link by 5 -moves (this is an easy application of the Jones polynomial evaluated at $t=e^{\pi i / 5}$ ). It is however an open problem whether any link can be changed to a trivial link by moves of the type shown in Fig. 2.3 [Nak] (it holds for links up to 7 crossings, in particular for the Borromean rings). More generally, it holds for algebraic knots (in the sense of Conway). 


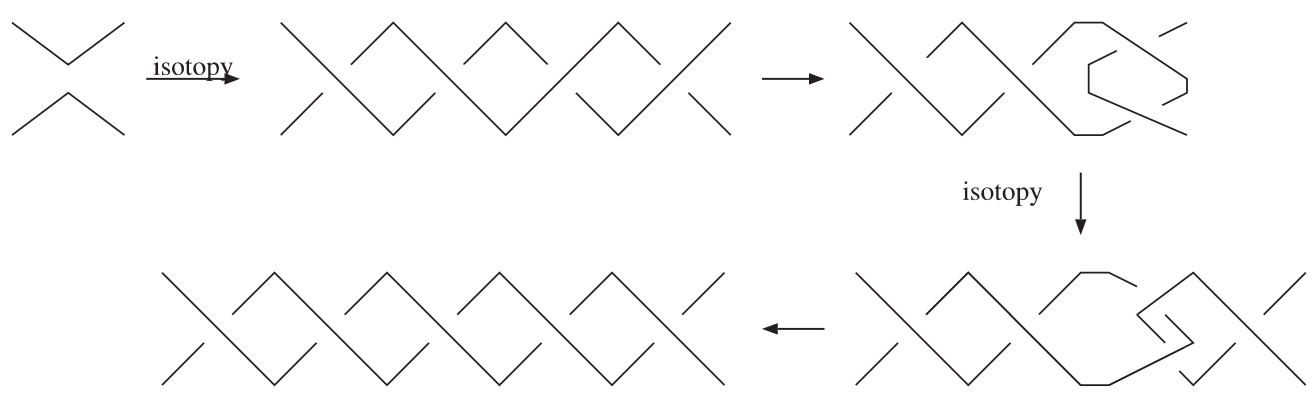

Fig. 2.4

The immediate generalization of the move of Fig. 2.3 is a move which changes $p$ horizontal half twists into $q$ vertical half twists. Let us call such a move (and its mirror image) a $[p, q]$-move; see Fig. 2.5.

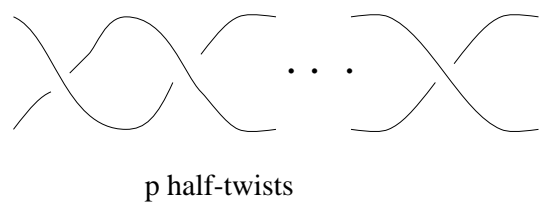

(p,q)-move

p half-twists

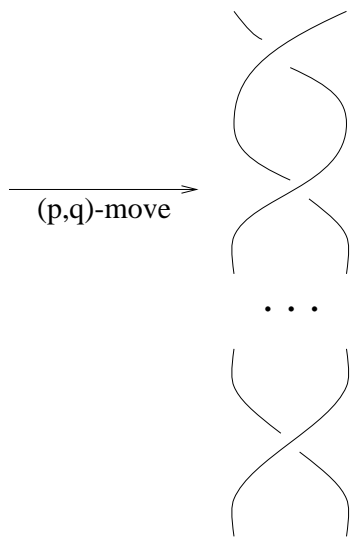

q half-twists

Fig. 2.5

EXERCISE 2.6. (a) Show that a $[p, q]$-move preserves the number of $(p q+1)$-colorings.

(b) Show that a $(2 p+1)$-move is a combination of a $[p, 2]$-move and a $[2, p]$-move. It is not always true that a $(p q+1)$-move is a composition of $[p, q]$-moves (and their inverses).

(c) Use linking numbers to show that if $p, q$ are odd numbers and $p+q$ is not a divisor of $p q+1$ (e. g. $p=q \geq 3$ ), then a $(p q+1)$-move is not a composition of $[p, q]$-moves (and their inverses). Show in particular that the torus link of type $(10,2)$ is not $[3,3]$ equivalent to the trivial link of 2 components (that is, the torus link cannot be obtained from the trivial link by the sequence of $[3,3]$-moves).

(d) Use the Kauffman polynomial to show that the 17 -move is not a composition of $[4,4]$-moves.

Hint to (d). Analyze how the Brandt-Lickorish-Millett polynomial, $Q_{L}(x)=F(1, x)$ changes under $[p, q]$-moves and $(p q+1)$-moves; compare [P-2]. Figure 2.6 illustrates the fact that a $[4,4]$ move preserves, up to the factor -1 , the $Q_{L}(x)$ polynomial modulo $x^{4}+x^{3}-2 x^{2}+1$. A 17 -move can change the polynomial $\left|Q_{L}(x)\right|$. 

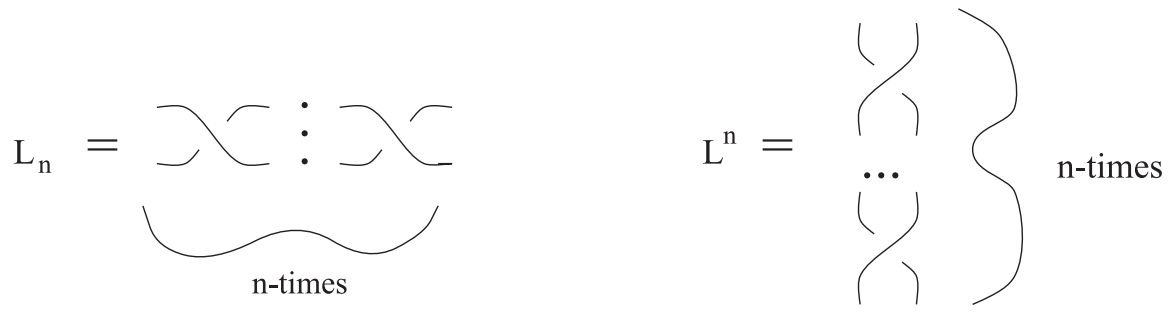

Fig. 2.6

Consider the Brandt-Lickorish-Millett polynomial of $L_{4}(x)$ and $L^{4}(x)$. From the relation $Q_{L_{n}}=x Q_{L_{n-1}}-Q_{L_{n-2}}+x Q_{L_{\infty}}$ one obtains

$$
Q_{L_{4}}=\left(x^{3}-2 x\right) Q_{L_{1}}-\left(x^{2}-1\right) Q_{L_{0}}+\left(x^{3}+x^{2}\right) Q_{L_{\infty}}{ }^{2},
$$

and

$$
\begin{aligned}
Q_{L^{4}} & =\left(x^{4}-3 x^{2}+1\right) Q_{L^{0}}-\left(x^{3}-2 x\right) Q_{L^{-1}}+\left(x^{4}+x^{3}-x^{2}\right) Q_{L^{\infty}} \\
& =\left(x^{4}-3 x^{2}+1\right) Q_{L_{\infty}}-\left(x^{3}-2 x\right) Q_{L_{1}}+\left(x^{4}+x^{3}-x^{2}\right) Q_{L_{0}} .
\end{aligned}
$$

Therefore for $x$ such that $x^{4}+x^{3}-2 x^{2}+1=0, Q_{L_{4}}=-Q_{L^{4}}$. On the other hand consider the torus link $L_{17,2}$, which can be reduced to the trivial link of 2 components by a 17-move. If a 17-move was the combination of $[4,4]$-moves then $Q_{L_{17,2}}(x)= \pm \frac{2-x}{x}$ for $x^{4}+x^{3}-2 x^{2}+1=0$. One can check however that $\frac{x}{2}\left(Q_{L_{17,2}}(x)+\frac{2-x}{x}\right)$ is an irreducible polynomial of degree equal to 17 and

$$
Q_{L_{17,2}}(x)-\frac{2-x}{x}=2 \frac{x-1}{x}\left(1-4 x-10 x^{2}+10 x^{3}+15 x^{4}-6 x^{5}-7 x^{6}+x^{7}+x^{8}\right)^{2},
$$

which for $x=q+q^{-1}$ gives

$$
2 \frac{q-1+q^{-1}}{q+q^{-1}} q^{-16}\left(\frac{q^{17}-1}{q-1}\right)^{2} .
$$

Thus $L_{17,2}$ is not $[4,4]$ equivalent to the trivial link of 2 components.

It is a nice exercise in linear algebra to show that the number of $n$-colorings is preserved by certain generalizations of n-moves.

Let $t_{\Delta, k}$ denote the righthanded half-twist performed on $k$ strings; see Fig. 2.7.

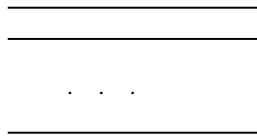

D

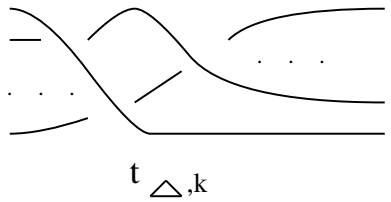

$\mathrm{t}_{\triangle, \mathrm{k}}$

Fig. 2.7

\footnotetext{
${ }^{2}$ It can be easily checked by induction, see [P-2], that generally $Q_{L_{n}}=U_{n-1} Q_{L_{1}}-U_{n-2} Q_{L_{0}}+$ $\frac{x}{x-2}\left(U_{n-1}-U_{n-2}-1\right) Q_{L_{\infty}}$ where $U_{i}(x)$ is the Chebyshev polynomial of the second type defined by: $U_{0}(x)=1, U_{1}(x)=x, U_{i}(x)=x U_{i-1}(x)-U_{i-2}(x)$.
} 
LEMMA 2.7. (a) $t_{\Delta, k}^{4}$ preserves $\operatorname{col}_{n}(D)$, for odd $k$ and any $n$.

(b) $t_{\Delta, k}^{2 n}$ preserves $\operatorname{col}_{2 n}(D)$, for an even $k$.

(c) Lemma 2.2(b) is stronger than (b) for $k=2$, and can be written as: $t_{\Delta, 2}^{n}$ preserves $\operatorname{col}_{n}(D)$.

3. Coloring and algebraic topology. It is useful to look at Lemma 2.2 from the point of view of algebraic topology.

DEFinition 3.1. Consider the abelian group of all colorings of arcs of a diagram using integers as colors. In other words consider the free abelian group spanned by all arcs of the diagram. Let each crossing give the relation: the sum of the colors of the undercrossings is equal to twice the color of the overcrossing. Let $H_{D}$ denote the group described.

LEMMA 3.2. (a) $H_{D}$ is preserved by Reidemeister moves, therefore it is a link invariant, $H_{L}$.

(b) $H_{D}$ reduced modulo $n$ (i.e. $H_{D} \otimes Z_{n}$ ) is the group of $n$-colorings of $D$.

THEOREM 3.3. $H_{L}$ is the direct sum of the first homology group of the cyclic branched double cover of $S^{3}$ with branching set $L$ and the infinite cyclic group. That is: $H_{L}=$ $H_{1}\left(\left(M_{L}\right)^{(2)}, Z\right) \oplus Z$.

Before we offer two proofs of the theorem we can carry our combinatorial construction one step further.

Definition 3.4 ([F-R]). $G_{D}$ is the group associated to the diagram $D$ as follows: generators of $G_{D}$ correspond to arcs of the diagram. Any crossing $v_{s}$ yields the relation $r_{s}=y_{i} y_{j}^{-1} y_{i} y_{k}^{-1}$ where $y_{i}$ corresponds to the overcrossing and $y_{j}, y_{k}$ correspond to the undercrossings at $v_{s}$.

The group $G_{D}$ was introduced by R. Fenn and C. Rourke [F-R] as an example of a rack's functor. They call it the associated core group of a link; compare with the core group of Joyce [Joy], an example of an involutory quandle. Joyce refers to the 1958 book of Bruck [Bruc] as the source of the idea; compare paragraphs 1, 2 and 19 of [Joy]. The topological interpretation of $G_{D}$ was given by M. Wada [Wa]; see Theorem 3.6.

LEMMA 3.5. (a) $G_{D}$ is preserved by Reidemeister moves, so it is a link invariant, $G_{L}$.

(b) The abelianization of $G_{D}$ yields $H_{D}$.

Lemma 3.5(b) and Theorem 3.3 suggest that $G_{D}$ may be related to the fundamental group of the branched 2 -fold cover over $D$. This is in fact the case.

THEOREM 3.6 ([Wa]). $G_{L}$ is the free product of the fundamental group of the cyclic branched double cover of $S^{3}$ with branching set $L$ and the infinite cyclic group. That is: $G_{L}=\pi_{1}\left(\left(M_{L}\right)^{(2)}\right) * Z$.

We will give later an elementary proof of the Wada theorem.

To prove Theorems 3.3 and 3.6, we need a combinatorial definition of another, well known, group.

DEFinition 3.7. $\Pi_{D}$ is the group associated to an oriented link diagram $D$ as follows: generators of the group, $x_{1}, \ldots, x_{n}$, correspond to arcs of the diagram; any crossing $v_{s}$ 
yields the relation $r_{s}=x_{i}^{-1} x_{j}^{-1} x_{i} x_{k}$ or $r_{s}=x_{i} x_{j} x_{i}^{-1} x_{k}^{-1}$, where $x_{i}$ corresponds to the overcrossing and $x_{j}, x_{k}$ correspond to the undercrossings at $v_{s}$ and the first relation comes from a positive crossing, Fig. 3.1(a), and the second comes from a negative crossing, Fig. 3.1(b).

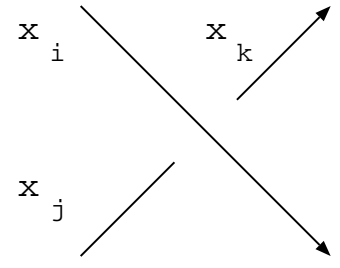

(a)

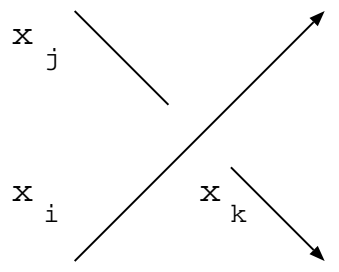

(b)

Fig. 3.1

LEMma 3.8. (a) $\Pi_{D}$ is preserved by Reidemeister moves, so it is a link invariant.

(b) The abelianization of the group $\Pi_{D}$ is a free group of $\operatorname{com}(D)$ generators.

(c) $\Pi_{D}$ does not depend on the orientation of the link. In particular if we change the orientation of a component, say $D_{1}$, of $D$ to get the diagram $D^{\prime}$, then the isomorphism of the group $\Pi_{D}$ generated by $\left(x_{1}, \ldots, x_{n}\right)$ onto the group $\Pi_{D^{\prime}}$ generated by $\left(x_{1}^{\prime}, \ldots, x_{n}^{\prime}\right)$ is given by sending $x_{i}$ to $x_{i}^{\prime}$ or $x_{i}^{\prime-1}$ depending on whether the arc of $x_{i}$ preserves or changes orientation when going from $D$ to $D^{\prime}$.

The group $\Pi_{D}$ and its presentation, which we described, was introduced by W. Wirtinger at his lecture delivered at a meeting of the German Mathematical Society in 1905 [Wi].

THEOREM 3.9 (Wirtinger). $\Pi_{D}$ is the fundamental group of the complement of the link; i.e. $\Pi_{D}=\pi_{1}\left(S^{3}-D\right)$.

We will not use this theorem, except for further algebraic-topological interpretations. For the proof see [C-F], [Rol], or [B-Z].

With the group $\Pi_{D}$ defined, we can give Fox's interpretation of $n$-colorings.

Let $D_{n}$ denote the dihedral group, i.e. the group of isometries of a regular $n$-gon. $D_{n}$ has a presentation: $D_{n}=\left\{\alpha, s: \alpha^{n}=1, s^{2}=1, s \alpha s=\alpha^{-1}\right\}$. The rotations, $\left\{\alpha^{k}\right\}$, form a cyclic subgroup, a $Z_{n}$. Reflections can be written as: $s_{k}=s \alpha^{k}$.

LEMMA 3.10. $n$-colorings of $D$ are in bijection with homomorphisms from $\Pi_{D}$ to $D_{n}$, which send $x_{i}$ to reflections. Namely, for an n-coloring $c$, the homomorphism $\phi_{c}: \Pi_{D} \rightarrow$ $D_{n}$ is given by: $\phi_{c}\left(x_{i}\right)=s_{k}$, where $k$ is the color of the arc which correspond to $x_{i}$.

To prove Theorem 3.3 we need still more preparation.

Let $\nu: \Pi_{D} \rightarrow Z_{2}$ be the modulo 2 evaluation map, that is, it sends words of even length (in the generators $x_{i}^{ \pm 1}$ ) to 0 , and words of odd length to one. $\nu$ is well defined because the relations of the group $\Pi_{D}$ have even length. Denote by $\Pi_{D}^{(2)}$ the kernel, $\operatorname{ker}(\nu)$, of the epimorphism $\nu$. This is a subgroup of index 2 in $\Pi_{D}$. From the point of view of 
algebraic topology it is the fundamental group of the 2-fold cyclic covering of $S^{3}-D$. That is, $\Pi_{D}^{(2)}=\pi_{1}\left(\left(S^{3}-D\right)^{(2)}\right)$. The abelianization of $\Pi_{D}^{(2)}$ is the first homology group of $\left(S^{3}-D\right)^{(2)}$.

Lemma 3.11. (a) For any $n$-coloring, c, one has $\phi_{c}^{-1}\left(Z_{n}\right)=\Pi_{D}^{(2)}$.

(b) Any homomorphism $\phi_{c}: \Pi_{D} \rightarrow D_{n}$ lifts uniquely to a homomorphism $\phi_{c}^{(2)}: \Pi_{D}^{(2)} \rightarrow$ $Z_{n}$. In particular $\phi_{c}^{(2)}\left(x_{i}^{2}\right)=0$.

(c) For any $n$-coloring, $c$, the following diagram is commutative:

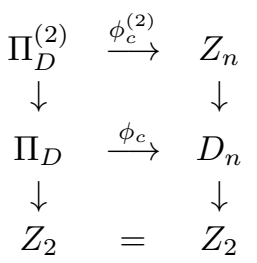

(d) Any homomorphism $\phi^{(2)}: \Pi_{D}^{(2)} \rightarrow Z_{n}$ such that $\phi^{(2)}\left(x_{i}^{2}\right)=0$ is a lift of exactly $n$ homomorphisms $\phi_{c}: \Pi_{D} \rightarrow D_{n}$.

Proof. (a) This is the case because rotations in $D_{n}$ (i.e. $Z_{n}$ ) are compositions of an even number of reflections.

(b) This follows from (a) and reflects the fact that $\phi_{c}$ sends words of even length to words of even length.

(c) This summarizes (a) and (b).

(d) Fix $x_{i}$. To show (d) it suffices to show that for any $s_{j}$, there is exactly one $c$ such that $\phi_{c}: \Pi_{D} \rightarrow D_{n}$ lifts to $\phi^{(2)}$ and $\phi_{c}\left(x_{i}\right)=s_{j}$. Namely we define $\phi_{c}(w)=\phi^{(2)}(w)$ if $w$ has an even length, and $\phi_{c}(w)=\phi^{(2)}\left(w x_{i}^{-1}\right) s_{j}$ if $w$ has an odd length. In particular $\phi_{c}\left(x_{k}\right)=$ $\phi^{(2)}\left(x_{k} x_{i}^{-1}\right) s_{j}$. We have to check that $\phi_{c}$ is a homomorphism. Consider $\phi_{c}\left(w_{1}\right) \phi_{c}\left(w_{2}\right)$. We have to check four cases, however for $w_{1}$ of even length the checking is immediate (e. g. if $w_{2}$ has odd length then $\phi_{c}\left(w_{1}\right) \phi_{c}\left(w_{2}\right)=\phi^{(2)}\left(w_{1}\right) \phi^{(2)}\left(w_{2} x_{i}^{-1}\right) s_{j}=\phi^{(2)}\left(w_{1} w_{2} x_{i}^{-1}\right) s_{j}=$ $\left.\phi_{c}\left(w_{1} w_{2}\right)\right)$. For the other cases we need to check first that $\phi^{(2)}(w w)=0$ for any $w$ of odd length.

We will show the slightly stronger fact that $w w$ lies in the commutator subgroup of the quotient group $\bar{\Pi}_{D}^{(2)}=\Pi_{D}^{(2)} /\left(x_{i}^{2}\right)$. Namely, let $w=x_{i_{1}} x_{i_{2}} \ldots x_{i_{2 m+1}}$. Then

$$
\begin{aligned}
w w & =x_{i_{1}} x_{i_{2}} \ldots x_{i_{2 m+1}} x_{i_{1}} x_{i_{2}} \ldots x_{i_{2 m+1}} \\
& =\left(x_{i_{1}} x_{1}\right)\left(x_{1} x_{i_{2}}\right) \ldots\left(x_{i_{2 m+1}} x_{1}\right)\left(x_{1} x_{i_{1}}\right) \ldots\left(x_{i_{2 m}} x_{1}\right)\left(x_{1} x_{i_{2 m+1}}\right) \\
& =\left(x_{i_{1}} x_{1}\right)\left(x_{i_{2}} x_{1}\right)^{-1}\left(x_{i_{3}} x_{1}\right)\left(x_{i_{4}} x_{1}\right)^{-1} \ldots\left(x_{i_{2 m+1}} x_{1}\right)\left(x_{i_{1}} x_{1}\right)^{-1} \ldots\left(x_{i_{2 m+1}} x_{1}\right)^{-1} .
\end{aligned}
$$

Now we can check that $\left.\phi_{c}\left(w_{1}\right) \phi_{c}\left(w_{2}\right)=\phi_{c}\left(w_{1} w_{2}\right)\right)$ for $w_{1}$ of odd length.

(i) If $w_{2}$ is of odd length then

$$
\begin{aligned}
\phi_{c}\left(w_{1}\right) \phi_{c}\left(w_{2}\right) & =\phi^{(2)}\left(w_{1} x_{i}^{-1}\right) s_{j} \phi^{(2)}\left(w_{2} x_{i}^{-1}\right) s_{j} \\
& =\phi^{(2)}\left(w_{1} x_{i}^{-1}\right)\left(\phi^{(2)}\left(w_{2} x_{i}^{-1}\right)\right)^{-1}=\phi^{(2)}\left(w_{1} x_{i}^{-1}\right) \phi^{(2)}\left(x_{i} w_{2}^{-1}\right) \\
& \left.=\phi^{(2)}\left(w_{1} w_{2}^{-1}\right)=\phi^{(2)}\left(w_{1} w_{2}\right) \phi^{(2)}\left(w_{2}^{-2}\right)=\phi_{c}\left(w_{1} w_{2}\right)\right) .
\end{aligned}
$$


(ii) If $w_{2}$ is of even length, then using (i) we get

$$
\begin{aligned}
\phi_{c}\left(w_{1}\right) \phi_{c}\left(w_{2}\right) & =\phi_{c}\left(w_{1}\right) \phi^{(2)}\left(w_{2} x_{i}^{-1} x_{i}\right)=\phi_{c}\left(w_{1}\right) \phi_{c}\left(w_{2} x_{i}^{-1}\right) \phi_{c}\left(x_{i}\right) \\
& =\phi^{(2)}\left(w_{1} w_{2} x_{1}^{-1}\right) s_{j}=\phi_{c}\left(w_{1} w_{2}\right) .
\end{aligned}
$$

The quotient group $\bar{\Pi}_{D}^{(2)}=\Pi_{D}^{(2)} /\left(x_{i}^{2}\right)$ can be interpreted as the fundamental group of the cyclic branched double cover of $S^{3}$ with branching set $D$; that is, $\bar{\Pi}_{D}^{(2)}=\pi_{1}\left(\left(M_{D}\right)^{(2)}\right)$. This interpretation follows from the fact that the elements $x_{i}^{2}$ correspond to meridians of boundary components of the unbranched double cover of $S^{3}-D$ and that these meridians are "killed" in the branched cover. The homomorphism $\phi^{(2)}$, from Lemma 3.11(d), factors through $\bar{\Pi}_{D}^{(2)}$, and because $Z_{n}$ is abelian, it factors through the abelianization of $\bar{\Pi}_{D}^{(2)}$. This abelianization can be interpreted as the first homology group of the cyclic branched double cover of $S^{3}$ with branching set $D$. We denote this group by $H_{1}=H_{1}\left(\left(M_{D}\right)^{(2)}, Z\right)$. Therefore we have the following commutative diagram:

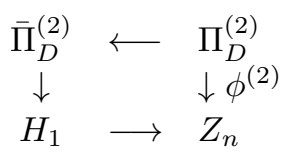

We have also a bijection between homomorphisms $H_{1} \rightarrow Z_{n}$, and homomorphisms $\phi^{(2)}: \Pi_{D}^{(2)} \rightarrow Z_{n}$ which satisfy condition (d) of Lemma 3.11. Thus Lemma 3.11 (d) leads to:

COROLlary 3.12. To any homomorphism $H_{1} \rightarrow Z_{n}$, there are uniquely associated $n$ different $n$-colorings. In particular the trivial homomorphism corresponds to $n$ trivial $n$-colorings. Therefore $H_{1} \otimes Z_{n} \oplus Z_{n}=\operatorname{Hom}\left(H_{1} \oplus Z_{n}, Z_{n}\right)$ has the same number of elements as $H_{D} \otimes Z_{n}$.

Because Corollary 3.12 holds for any $n$, we have $H_{D}=H_{1} \oplus Z$ and the proof of Theorem 3.3 is complete.

LEMMA 3.13. Let $F=\left\{x_{1}, \ldots, x_{n}:\right\}$ be the free group on $n$ generators. Let $F^{(2)}$ be its subgroup generated by the words of even length and let $\bar{F}^{(2)}$ be the quotient group $F^{(2)} /\left(x_{i}^{2}\right)$. Then:

(a) $F^{(2)}$ is a free group on $2 n-1$ generators $x_{1} x_{k}, x_{2} x_{k}, \ldots, x_{k-1} x_{k}, x_{k+1} x_{k}, \ldots, x_{n} x_{k}$, $x_{1}^{2}, x_{2}^{2}, \ldots, x_{n}^{2}$, where $x_{k}$ is any fixed generator of $F$.

(b) $\bar{F}^{(2)}$ is a free group on $n-1$ generators $x_{1} x_{k}, x_{2} x_{k}, \ldots, x_{k-1} x_{k}, x_{k+1} x_{k}, \ldots, x_{n} x_{k}$.

The above lemma is the starting point of our proof, given below, of the Wada theorem (3.6)

Proof of Theorem 3.6. Let $D^{\prime}$ denote the diagram obtained from the diagram $D$ by adding one trivial component.

STEP 1. $\bar{\Pi}_{D^{\prime}}^{(2)}=\bar{\Pi}_{D}^{(2)} * Z$.

Pr o of. Denote by $x_{1}, \ldots, x_{n}$ generators corresponding to $\operatorname{arcs}$ of the diagram $D$, and by $x_{n+1}$ the generator corresponding to the additional component of $D^{\prime} . \bar{\Pi}_{D^{\prime}}^{(2)}$ is generated by $x_{2} x_{1}, x_{3} x_{1}, \ldots, x_{n} x_{1}, x_{n+1} x_{1}$. Relations of the group are associated to crossings of the diagram $D^{\prime}$ (so $D$ ). The general relation is of the form $x_{i}^{-1} x_{j} x_{i} x_{k}^{-1}$ or $x_{i} x_{j} x_{i}^{-1} x_{k}^{-1}$ 
$(i, j, k \leq n)$; Fig. 3.1. Both relations lie in $F^{(2)}$ and in $\bar{F}^{(2)}$ they are both conjugate to $\left(x_{i} x_{1}\right)\left(x_{j} x_{1}\right)^{-1}\left(x_{i} x_{1}\right)\left(x_{k} x_{1}\right)^{-1} . \bar{\Pi}_{D^{\prime}}^{(2)}$ is a quotient of $\bar{F}^{(2)}$ by these relations (compare Lemma 3.14). No relation uses the generator $\left(x_{n+1} x_{1}\right)$. Therefore $\bar{\Pi}_{D^{\prime}}^{(2)}=\bar{\Pi}_{D}^{(2)} * Z$.

STEP 2. Consider the generators $x_{1} x_{n+1}, x_{2} x_{n+1}, \ldots x_{n} x_{n+1}$ of $\bar{\Pi}_{D^{\prime}}^{(2)}$. We can associate them to $\operatorname{arcs}$ of $D$ as follows: $x_{i} x_{n+1}$ corresponds to the $\operatorname{arc}$ of $D$ which before was associated to $x_{i}$. No generator corresponds to the additional arc of $D^{\prime}$. Relations associated to crossings of $D$ can be found as in Step 1 to be $\left(x_{i} x_{n+1}\right)\left(x_{j} x_{n+1}\right)^{-1}\left(x_{i} x_{n+1}\right)\left(x_{k} x_{n+1}\right)^{-1}$, where $i, j, k \leq n$. If we put $y_{s}=x_{s} x_{n+1}$ for $s \leq n$ then the relations reduce to $y_{i} y_{j}^{-1} y_{i} y_{k}^{-1}$. We get exactly the presentation of the group $G_{D}$ from Definition 3.4.Therefore $G_{D}=\bar{\Pi}_{D^{\prime}}^{(2)}=\bar{\Pi}_{D}^{(2)} * Z$. The proof of the Wada theorem is complete.

One can describe the group $\Pi_{D}^{(2)}$ similarly to the group $\bar{\Pi}_{D}^{(2)}$. A more challenging exercise is to find the Wirtinger type presentation of the fundamental group of the general $k$-fold cyclic branched cover of $S^{3}$ with branching set $D$, i.e. $\Pi_{D}^{(k)}=\pi_{1}\left(M^{(k)}\right)$. We describe the result below (compare [B-Z; Ch. 4]).

Theorem 3.14. (a) Let $F=F_{n+1}=\left\{x_{1}, x_{2}, \ldots x_{n+1}:\right\}$ and let $F^{(k)}$ be the kernel of the map $F \rightarrow Z_{k}$ which sends $x_{i}$ to 1 . Furthermore let $F^{(\infty)}=\operatorname{ker}(F \rightarrow Z)$. Define $\bar{F}^{(k)}=F^{(k)} /\left(x_{i}^{k}\right)$ and $y_{i}=x_{i} x_{n+1}^{-1}$. Let $\tau: F \rightarrow F$ be an automorphism given by $\tau(w)=x_{n+1} w x_{n+1}^{-1}$.

(i) $F^{(k)}$ is a free group generated freely by $n k+1$ elements $\tau^{j}\left(y_{i}\right)$, for $i \leq n$ and $0 \leq j \leq k-1$, and $x_{n+1}^{k}$.

(ii) $F^{(\infty)}$ is freely generated by elements $\tau^{j}\left(y_{i}\right)$, for $i \leq n$ and any integer $j$.

(iii) $\bar{F}^{(k)}$ is freely generated by $n(k-1)$ elements $\tau^{j}\left(y_{i}\right)$, for $i \leq n$ and $0 \leq j<k-1$. Notice that one has relations $y_{i} \tau\left(y_{i}\right) \ldots \tau^{k-1}\left(y_{i}\right)=1$, for any $i$.

(b) (i) $\Pi_{D}^{(k)} * \underbrace{Z * Z * \ldots * Z}_{k-1 \text { times }}=\Pi_{D \sqcup O}^{(k)}$ has the following Wirtinger type description:

There are $k-1$ generators, $\tau^{j}\left(y_{i}\right), 0 \leq j<k-1$, corresponding to the ith arc of the diagram $D$, and there are $k-1$ relations $\left.\tau^{j}\left(r_{s}\right)\right), 0 \leq j<k-1$, corresponding to any crossing, $v_{s}$, where $r_{s}$ depends on the sign of a crossing as follows:

$(+)$ In the case of the positive crossing (Fig. 3.1(a)): $r_{s}=y_{i} \tau\left(y_{k}\right)\left(\tau\left(y_{i}\right)\right)^{-1} y_{j}^{-1}$. (-) In the case of the negative crossing (Fig. 3.1(b)): $r_{s}=y_{i} \tau\left(y_{j}\right)\left(\tau\left(y_{i}\right)\right)^{-1} y_{k}^{-1}$. We have to remember that $\tau^{k-1}\left(y_{i}\right)=\left(y_{i} \tau\left(y_{i}\right) \ldots \tau^{k-2}\left(y_{i}\right)\right)^{-1}$.

(ii) $\Pi_{D}^{(\infty)} * F_{\infty}=\left\{\tau^{j}\left(y_{i}\right), i \leq n: \tau^{j}\left(r_{s}\right), j \in Z\right\}$, where $F_{\infty}$ is a countably generated free group.

Corollary 3.15. (i) $H_{1}\left(M^{(\infty)}\right) \oplus Z\left[t^{ \pm 1}\right]=Z\left[t^{ \pm 1}\right]\left(y_{1}, y_{2}, \ldots, y_{n}\right) /\left(\bar{r}_{s}\right)$, where $\bar{r}_{s}$ are relations associated to crossings: $\left(1-t^{\epsilon}\right) y_{i}+t^{\epsilon} y_{k}-y_{j}=0$, where $\epsilon= \pm 1$ is the sign of the crossing $v_{s}$; Fig. $3.1^{3}$.

(ii) $H_{1}\left(M^{(k)}, Z\right) \oplus(Z)^{k-1}=Z\left[t^{ \pm 1}\right] /\left(1+t+\ldots t^{k-1}\right)\left(y_{1}, y_{2}, \ldots, y_{n}\right) /\left(\bar{r}_{s}\right)$.

\footnotetext{
${ }^{3}$ We can think of it as Wirtinger type description of the Burau representation. Compare also [Re-1, Ch. II(14)].
} 
(iii) $H_{1}\left(M^{(k)}, Z_{m}\right) \oplus\left(Z_{m}\right)^{k-1}$ has the following "coloring" description: Every arc of the diagram is colored by a sequence, $\left(a_{0}, a_{1}, \ldots, a_{k-2}\right)$, of colors taken from the set of $m$ colors, $(0,1,2, \ldots, m-1)$. These colorings form a $Z_{m}$-module $Z_{m}^{\lambda(k-1)}$, where $\lambda$ is the number of arcs in the diagram. Coloring of an arc can be coded by a polynomial of degree $k-2$ with coefficients in $Z_{m}, w=\sum_{i=0}^{k-2} a_{i} t^{i}$. Now we consider the space (submodule) of allowed coloring, that is, colorings which at any crossing satisfy the equation: $\left(1-t^{\epsilon}\right) w_{i}+$ $t^{\epsilon} w_{k}-w_{j}=0$, where $1+t+\ldots t^{k-1}=0\left(\right.$ in particular $\left.t^{-1}=-1-t-t^{2}-\ldots-t^{k-2}\right), \epsilon= \pm 1$ as in $(i), w_{l}$ are polynomials of degree $k-2$ with coefficients in $Z_{m}$ corresponding to arcs at a crossing as in Fig. 3.1. Allowed colorings form a group $H_{1}\left(M^{(k)}, Z_{m}\right) \oplus\left(Z_{m}\right)^{k-1}$, compare $[\mathrm{S}-\mathrm{W}]$.

We can generalize the group $H_{D}$ in yet another direction. We can consider the $|a|$ by $|v|$ matrix $\left(b_{i, j}\right)$ where $|a|$ is the number of arcs of the diagram and $|v|$ the number of crossings of the diagram, and where $b_{i, j}=0$ if the $i$ th arc is disjoint from the $j$ th crossing, $b_{i, j}=2$ if the $i$ th arc is the overcrossing of the $j$ th crossing and $b_{i, j}=-1$ if the $i$ th arc is an undercrossing of the $j$ th crossing. We consider the matrix up to changes caused by Reidemeister moves. Virtually nothing is known about the invariant given by this matrix (except the group $H_{D}$ ). One should, at least, compare this matrix with the Goeritz matrix and the Seifert matrix (see [Gor]).

4. Coloring and statistical mechanics. Further modification of our method leads to state models of statistical mechanics and the Yang-Baxter equation. We will illustrate it by two examples. In the first we consider the state sum corresponding to $n$-colorings and in the second we give (after Jones) the state sum approach to the skein (generalized Jones) polynomial.

EXAMPLE 4.1. For $n$ colors, $0,1, \ldots, n-1$, every coloring of arcs of a diagram by these colors, is called a state of the diagram. We associate to any state, $s$, and any crossing $v$, a weight, $w(v, s)$, depending on the colors of arcs at $v$. For Fox colorings it will be 1 if twice the color of the overcrossing is congruent to the sum of the colors of the undercrossings modulo $n$. It will be 0 otherwise. We associate to any state, $s$, the global weight equal to $\Pi_{v} w(v, s)$. For a Fox coloring it is 1 if $s$ is an $n$-coloring and 0 otherwise. Finally we define the partition function, $Z_{D}(n)$, to be the sum over all states of their global weights, i.e.

$$
Z_{D}(n)=\sum_{s} \prod_{v} w(v, s)
$$

In our example we get $Z_{D}(n)=\operatorname{col}_{n}(D)$. This state sum description of Fox n-colorings was given in [H-J], and it is called, in statistical mechanics, a vertex type model.

In our example, colors were associated to arcs of the diagram; in the general case of the vertex model weights are associated to edges of a graph. So we have to think of the link diagram as a graph with additional structure (under-over crossing) at the vertices.

EXAMPLE 4.2. Let a link diagram be considered as a graph with vertices of valency 4 at crossings and vertices of valency 2 at maxima and minima. We assume that there are 
only a finite number of extrema and that crossings are positioned vertically, so that after smoothing them, one do not introduce new extrema. A state is a function from edges of the diagram to the set of $k$ colors. We consider weights to be in $Z\left[q^{ \pm 1}\right]$. With any vertex, $v$, of degree 4 , and inputs with colors $i, j$ and output with colors $k, l$, we associate the weight

$$
w_{+}(i, j ; k, l)= \begin{cases}q-q^{-1} & \text { if } i<j, i=k, j=l, \\ 1 & \text { if } i=l, j=k, i \neq j, \\ q & \text { if } i=j=k=l, \\ 0 & \text { otherwise, }\end{cases}
$$

in the case of a positive crossing, and

$$
w_{-}(i, j ; k, l)= \begin{cases}q^{-1}-q & \text { if } i>j, i=k, j=l, \\ 1 & \text { if } i=l, j=k, i \neq j, \\ q^{-1} & \text { if } i=j=k=l, \\ 0 & \text { otherwise, }\end{cases}
$$

in the case of a negative crossing; see Fig. 4.1.

For any vertex of degree 2 , we associate the weight $w(i)=q^{ \pm 1 / 2(2 i-k-1)}$, according to the convention of Fig. 4.2.

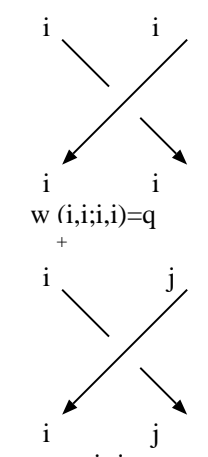

$\mathrm{i}<\mathrm{j}$
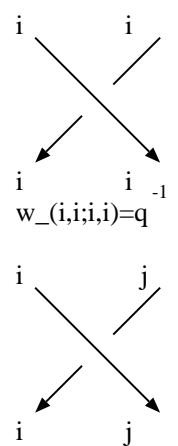

$\mathrm{i}<\mathrm{j}$

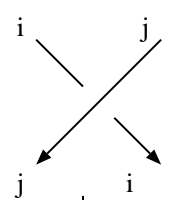

$i \neq j$

$w(i, j ; j, i)=w(i, j \cdot j, i)$

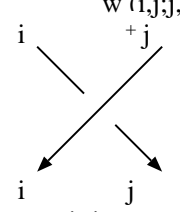

$\mathrm{i}>\mathrm{j}$
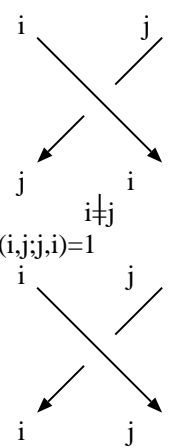

$\mathrm{i}>\mathrm{j}$

$w_{+}(i, j ; i, j)=0 \quad$ w_ $(i, j ; i, j)=q^{-1}-q$

Fig. 4.1
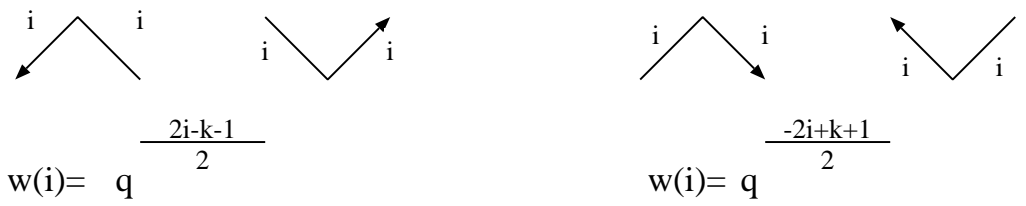

$w(i)=q^{\frac{-2 i+k+1}{2}}$

Fig. 4.2

As proven by Jones [Jo-1] the partition function given by this vertex model is equal to the version of the skein (homflypt) polynomial for regular isotopy classes of links (associated with the skein relation $\left.q^{k} P_{L_{+}}-q^{-k} P_{L_{-}}=\left(q-q^{-1}\right) P_{L_{0}}\right)$. 


\section{References}

[A-B] J. W. Alexander, G. B. Briggs, On types of knotted curves, Ann. of Math 28 (1926/27), 562-586.

[Bruc] R. H. Bruck, A survey of binary systems, Springer, Berlin 1958.

[Br] H. Brunn, Topologische Betrachtungen, Zeitschrift für Mathematik und Physik, 37 (1892), 106-116.

[B-Z] G. Burde, H. Zieschang, Knots, De Gruyter (1985).

[C-F] R. H. Crowell, R. H. Fox, An introduction to knot theory, Ginn and Co., 1963.

[Cox] H. S. M. Coxeter, Factor groups of the braid group, Proc. Fourth Canadian Math. Congress, Banff, 1957, 95-122.

[F-R] R. Fenn, C. Rourke, Racks and links in codimension two, Journal of Knot Theory and its Ramifications, 1(4) 1992, 343-406.

[F-1] R. H. Fox, A quick trip through knot theory, In: Top. 3-manifolds, Proc. 1961 Top. Inst. Univ. Georgia (ed. M. K. Fort, jr), 120-167. Englewood Cliffs. N. J. : PrincetonHall, 1962.

[F-2] R. H. Fox, Metacyclic invariants of knots and links, Canadian J. Math., XXII(2) 1970, 193-201.

[Ga] C. F. Gauss, Zur mathematischen Theorie der electrodynamischen Wirkungen, 1833, Werke, Königliche Gesellschaft der Wissenschaften zu Göttingen, 5 (1877), 602-629.

[G-J] D. Goldschmidt, V. F. R. Jones, Metaplectic link invariants, Geometriae Dedicata, 31 (1989), 165-191.

[Goe] L. Goeritz, Knoten und quadratische Formen, Math. Z., 36(1933), 647-654.

[Gor] C. McA. Gordon, Some aspects of classical knot theory, In: Knot theory, L. N. M. 685 (1978) 1-60.

[H-U] T. Harikae, Y. Uchida, Irregular dihedral branched coverings of knots, in Topics in knot theory, N. A. T. O. A. S. I. series C, 399, (ed. M. Bozhüyük) Kluwer Academic Publisher (1993), 269-276.

[H-J] P. de la Harpe, V. F. R. Jones, Graph invariants related to statistical mechanical models: Examples and Problems, J. Combinat. Theory B., 57, 1993, 207-227.

[J-P] W. Jakobsche, J. H. Przytycki, Topologia 3-wymiarowych rozmaitości, Wydawnictwa Uniwersytetu Warszawskiego, 1987.

[Ja] F. Jaeger, Composition products and models for the Homfly polynomial, L'Enseignement Mathématique, 35 (1989), 323-361.

[Ja-P] F. Jaeger, J. H. Przytycki, A non-commutative version of the Goeritz matrix of a link, in preparation.

[Jo-1] V. F. R. Jones, On knot invariants related to some statistical mechanics models, Pacific J. Math., 137(2), 1989, 311-334.

[Jo-2] V. F. R. Jones, Subfactors and knots, CBMS, Regional Conference Series in Mathematics 80, AMS 1991.

[Joy] D. Joyce, A classifying invariant of knots: the knot quandle, Jour. Pure Appl. Alg., 23 (1982), 37-65.

[K] L. H. Kauffman, Knots and Physics, Series on Knots and Everything - Vol. 1, World Scientific, 1991.

[Li] W. B. R. Lickorish, Polynomials for links. Bull. London Math. Soc. 20 (1988) 558588. 
[L-M] W. B. R. Lickorish, K. Millett, Some evaluations of link polynomials, Comment. Math. Helv., 61(1986), 349-359.

[Liv] C. Livingston, Knot theory, The Carus Math. Monographs, Vol 24, MAA 1993.

[Mon] J. M. Montesinos, Lectures on 3-fold simple coverings and 3-manifolds, Contemporary Mathematics 44 (Combinatorial methods in topology and algebraic geometry), 1985, 157-177.

[Mo] H. R. Morton, Problems, in Braids, Ed. J. S. Birman, A. Libgober, AMS Contemporary Math., 78(1988), 557-574.

[Mur] H. Murakami, Unknotting number and polynomial invariants of a link, preprint 1985.

[Nak] Y. Nakanishi, On generalized unknotting operations, J. Knot Theory and its Ramifications, 3(2), 1994, 197-209.

[P-1] J. H. Przytycki, Elementary conjectures in classical knot theory, in Quantum Topology, Ed. L. J. Kauffman, R. A. Baadhio, Series on Knots and Everything - Vol. 3, World Scientific, 1993, 292-320.

[P-2] J. H. Przytycki, $t_{k}$-moves on links, In Braids, ed. J. S. Birman and A. Libgober, Contemporary Math. Vol. 78 (1988) 615-656.

[Re] K. Reidemeister, Elementare Begründung der Knotentheorie, Abh. Math. Sem. Univ. Hamburg, 5 (1927), 24-32.

[Re-1] K. Reidemeister, Knotentheorie. Ergebn. Math. Grenzgeb., Bd. 1; Berlin: SpringerVerlag (1932) (English translation: Knot theory, BSC Associates, Moscow, Idaho, USA, 1983).

[Rol] D. Rolfsen, Knots and links, Publish or Perish, 1976.

[R-T-1] N. Reshetikhin, V. Turaev, Ribbon graphs and their invariants derived from quantum groups, Jour. Commun. Math. Phys., 127 (1990), 1-26.

[R-T-2] N. Y. Reshetikhin, V. Turaev, Invariants of three manifolds via link polynomials and quantum groups. Invent. Math. 103 (1991) 547-597.

[S-W] D. Silver, S. G. Williams, Generalized n-Colorings of Links, this volume.

[Tu-1] V. G. Turaev, The Yang-Baxter equation and invariants of links, Invent. Math., 92(1988), 527-553

[T-V] V. G. Turaev, O. Y. Viro, State sum invariants of 3-manifolds and quantum 6jsymbols, Topology, 31 (1992), 865-902.

[Wa] M. Wada, Group invariants of links, Topology 31(2) 1992.

[Wi] W. Wirtinger, Über die Verzweigungen bei Funktionen von zwei Veränderlichen, Jahresbericht d. Deutschen Mathematiker Vereinigung, 14 (1905), 517. (The title of the talk supposedly given at September 261905 at the annual meeting of the German Mathematical Society in Meran).

[Wu $\mathrm{F} . \mathrm{Y}$. Wu, Knot theory and statistical mechanics, Review of modern physics, 64(4), October 1992, 1099-1131. 